\title{
Failure Modes and Mechanical Properties of Bracket Anchor Connections for Cross-Laminated-Timber
}

\author{
Shen Yinlan ${ }^{1,2 *}$, Johannes Schneider ${ }^{3}$, Siegfried F. Stiemer ${ }^{3}$, Ren Xueyong $^{4}$ \\ 1The Key Laboratory of Urban Security and Disaster Engineering of Ministry of Education, Beijing University of Technology 100124 , \\ China \\ 2 Beijing Key Lab of Earthquake Engineering and Structurall Retrofit, Beijing University of Technology 100124, China \\ 3Dept. of Civil Engineering, Applied Science, University of British Columbia,BC V6T 1Z4, Canada \\ 4MOE Key Laboratory of Wooden Material Science and Application, College of Materials Science and Technology, Beijing Forestry \\ University, Beijing 100083, China
}

\begin{abstract}
The paper presents some experimental data and phenomenon on bracket anchor connections for Cross-Laminated-Timber. The goal of this research is to provide a better understanding of the seismic performance of bracket connections subjected to seismic actions and how to choose and design bracket connections for Cross-Laminated-Timber structures. Test configuration and experimental setups are illustrated in details; cyclic displacement schedules of the connections in two directions are presented considering that CLT wall has horizontal sliding in the plane and uplift at the end in quasi-state tests. Different failure modes and force transmission mechanisms of different connections under the loading protocol were analysed. And important quantities for seismic design such as strength, and stiffness, equivalent yield load, peak load and ductility of the connections are evaluated and compared among different kinds of connections; an excellent connector is revealed in ductility and load capacity by test data analysis. In addition, some suggestions to choose and design bracket anchor connections are given.
\end{abstract}

\section{INTRODUCTION}

Cross Laminated Timber (abbreviation CLT), as a new generation of engineered wood product, is already well-established in Europe, and recently is also gaining popularity in North America, Australian and Asian market. Ceccotti and Follisa(2006) $)^{[1]}$ in the SOFIE research project, Dujic etal. (2004) ${ }^{[2]}$ in Slovenia and Popovski etal. (2010) ${ }^{[3]}$ in FPInnovation of Canada carried out a series of monotonic and cyclic loading tests on CLT shear walls with different size and different types of anchor connections. These tests showed that the CLT panels are relatively stiff and the connections between the base and the panel are sources of ductility and energy dissipation (hence the performances are controlled by the connections). Fragiacomo etal. $(2011)^{[4]}$ revealed that different methods to model connections between panels in CLT buildings can markedly influence the building stiffness, vibration period, base shear forces and building ductility. Shen etal.(2013) ${ }^{[5]}$ developed numerical models for the connections with consideration of most hysteretic characteristics(including pinching behavior, strength and stiffness degradation, rapid degradation of reloading stiffness and unloading stiffness) and demonstrated that the CLT wall model calibrated based on connection level is valid to predict the pushover and hysteretic response of the full-size wall tests. The most important component is the mechanical connections between adjacent panels and with the foundations which affect the seismic performance of CLT buildings, as most of the building flexibility is concentrated in the connections. In addition, mechanical connections in CLT buildings play an important role in maintaining integrity of building structures and providing the necessary strength, stiffness, stability and ductility ${ }^{[4]}$.Thus, understanding the mechanical behavior and failure modes of connections in CLT buildings is crucial for their seismic design. Experimental values are also needed to feed advanced, for further simplified numerical models and damage assessments for seismic analysis of CLT Structures.

Metal angle bracket connections, hold-down connections, screws connections and dowel type connections are commonly used to transfer forces in CLT buildings. And metal angle bracket connections are most used to connect wall to floors and wall to foundation in order to resist bigger shear and tension forces in wall panels caused by wind or by a seismic event. The current relative research is focus on the analysis of mechanical properties from experimental angle connection tests ${ }^{[6,7]}$, analytical models (Uibel and Bla $\beta$ ' method, formulas in Eurocode 5) calculation comparison with experimental values ${ }^{[7]}$ and the invention of innovative connections ${ }^{[8-10]}$. Gavic and Fragiacomo $^{[7]}$ pointed that analytical formulae 
overestimate the experimental values, and recommended that experimental values of angle bracket connections are necessary. The study of failure modes is lack of investigation, and force transmission mechanisms with different failure modes are not clear. In addition, there are no standards or references about how to choose and design the connections for CLT buildings seismic design.

In this paper, experimental results and phenomenon of the angle anchor bracket connections are presented and critically discussed. Test configuration, experimental setups and loading protocol are illustrated in details; different failure modes and force transmission mechanisms of different kinds of connections are analyzed. Important quantities for seismic design are evaluated and compared among a series of different connections. Meanwhile, an excellent connector in ductility and load capacity is revealed by test data analysis. Lastly, some suggestions to choose and design angle anchor connections are given.

\section{EXPERIMENTAL PROGRAMME}

\subsection{Test configurations and setups}

The test specimens consist of three elements: Brackets, fasteners and CLT blocks. Three types of brackets are used in the connection tests according to their shear and tension capacities and construction requirements.

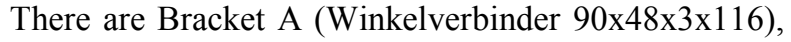
Bracket B (BMF Winkelverbinder 105) and Bracket C (AKR135LG-B-Winkelverbinder) with the materials of S250GD+Z275, produced by SIMPSON StrongTie Company (Fig.1). A typical bracket is attached with one bracket leg to the CLT panel and the other leg is bolted to the foundation with various anchor systems. The major difference between the brackets is the number of possible fasteners and the distance between the vertical leg of the bracket and the holes for the anchor bolts.

The principle of choosing fasteners is based on ductility and resisting slipping capacity. There are three kinds of fasteners including Spiral nail 16d x 3 1/2", Screw 5 x 90mm and Screw 4 x 70mm (Fig.2).

CLT blocks were 3-layers with a thickness of 94mm (30-34-30) provided by KLH Company. In addition, the experimental CLT materials meet the requirement of Grade E3 from APA performance-rated CLT Standards ${ }^{[11]}$. In shear wall tests there are in general two directions of movements (including horizontal sliding in the plane of the wall and uplift at the end of the wall). To measure the deformation and load-slip capacity in parallel and perpendicular to the outer grain of CLT, two different test set-ups were required and two different sizes of CLT blocks were produced. The target of connection tests is that the combination variety between brackets and fasteners increases the amount of testing required to find the optimal fasteners with the ideal properties and failure behavior.

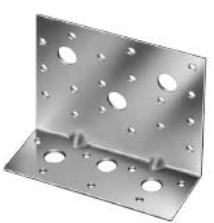

(a)

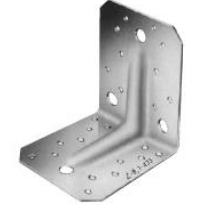

(b)

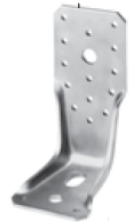

(c)
Fig 1. Brackets:(a) A;(b) B;(c) C

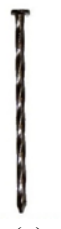

(a)

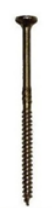

(b)

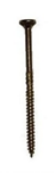

(c)
Fig.2. Fasteners: (a) Spiral nail 16d x 3 1/2";(b) Screw 5 x 90mm;(c) Screw 4 x 70mm

All connection tests were done using an INSTRON8802 testing machine with a maximum capacity in compression of $250 \mathrm{kN}$ and total mechanical travel of $150 \mathrm{~mm}$. Figure 3 illustrates the first test setup for testing connections parallel to the outside layered grain. The top end was connected to the testing apparatus by friction and shear using steel clamping plates and 1/2" bolts. A typical anchor angle bracket is attached with one bracket leg to the CLT panel and the other leg is bolted to the thick steel plate with the thickness of $40 \mathrm{~mm}$ as foundation platform anchored on the actuator head. It should be noted that due to Lshape bracket on one side of adjacent members, nonaxial force and eccentric bending moment are created. Therefore, the steel roller is installed on the back of CLT panel in order to provide the resistance but have no effect on the vertical movement of the specimen.

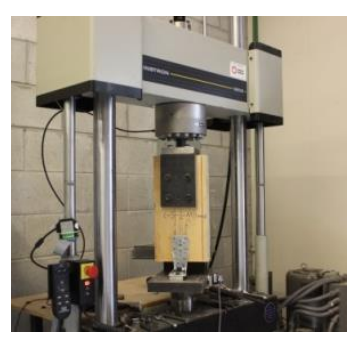

Fig.3. Connection test parallel to outer grain

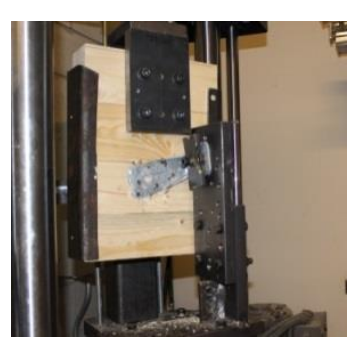

Fig.4 Connection test perpendicular to outer grain
Figure 4 illustrates the second test set-up for testing connections perpendicular to the outside layered grain. Unlike testing parallel to grain, the bracket in this set-up was attached with one leg to the CLT panel and the other leg is bolted to a vertical side of L-shape thick steel plate(hence a horizontal side is connected rigidly to the load cell). It should be noted that the steel roller is installed on the back of CLT panel in the same way and U-shaped steel plates are designed on both sides of a CLT panel for avoiding out-plane buckling. The data of applied load, movement of the actuator 
head, and relative displacement of the connection with respect to the foundation platform are collected during the tests.

\subsection{Loading protocol choose}

There is no particular standard for CLT shear wall or CLT connections. The ASTM is the most recognized code in North America as Popovski etal. (2010) ${ }^{[3]}$ in FPInnovation of Canada carried out a series of CLT wall tests with different sizes and different types of anchor connections according to ASTM-CUREE ${ }^{[12]}$.

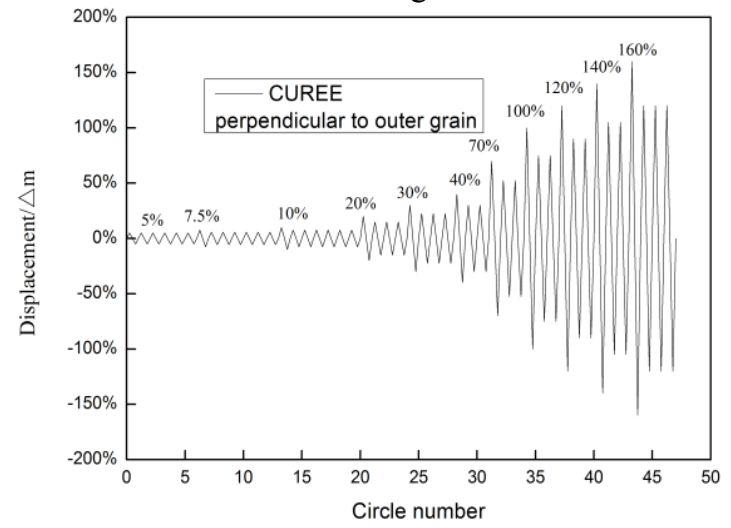

Fig.5(a).CUREE(perpendicular to outer grain)

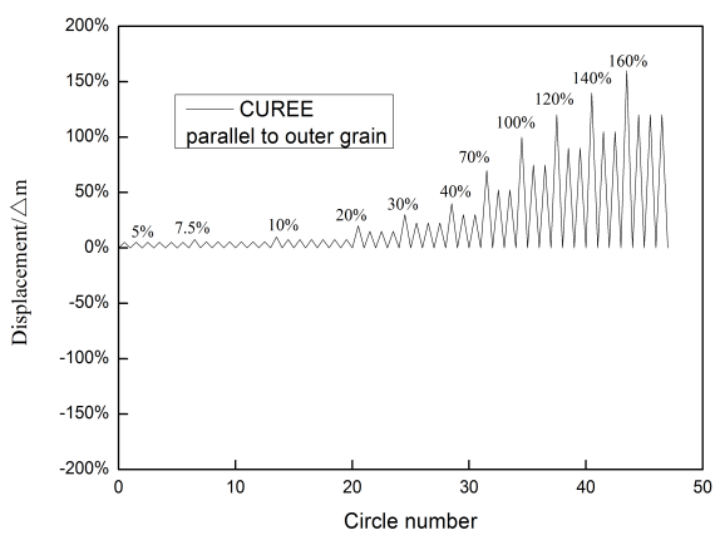

Fig. 5(b). CUREE(parallel to outer grain)

ASTM-CUREE loading procedure consists of initiation cycles at the beginning, primary cycles with amplitude expressed as a fraction (percent) of the reference deformation, and trailing cycles followed with the amplitude that is equal to $75 \%$ of the amplitude of the preceding primary cycle. Krawinkler $^{[13]}$ showed that this loading history is to evaluate capacity level seismic performance of components subjected to ordinary ground motions whose probability of exceedance in 50 years is 10 percent. The cyclic displacement schedules in two directions are presented in Fig. 5(a) and 5(b), considering that CLT panel subjected to quasi-state monotonic and cyclic loading has horizontal sliding in the plane of the wall and uplift at the end of the wall.

\section{EXPERIMENTAL TEST RESULTS AND DISCUSSION}

\section{1 failure mode analysis}

In preliminary tests, various combinations of brackets and fasteners were tested under monotonic parallel and perpendicular to grain set-up. There are five failure modes occurred and failure modes analysis are carried on in the following.

Failure mode(a): At the beginning, the bracket coordinate fasteners embedded in the CLT block to do elastic motions and fasteners are subjected to tension or shear force by the bracket; with increasing deformation controlled quasi-static, fasteners begin to have plastic deformation with forming plastic hinges; at the same time, there are contact boundary to crimp or separate between fasteners and CLT block; afterwards, a cavity around the fastener is formed by wood crushing, which lead to slackness or loosening of fasteners embedded in the CLT materials; finally, bond strength and friction are not enough to fix the place of the fasteners and fasteners are pulled out from CLT block. Pull-out failure is the ideal failure mode as the most energy can be dissipated by forming plastic hinges while not destroying the CLT block. It is crucial that not to damage the primary structure (CLT) of the building during the earthquake.

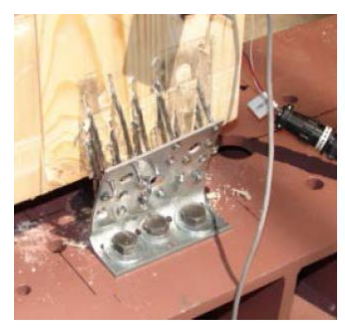

(a)

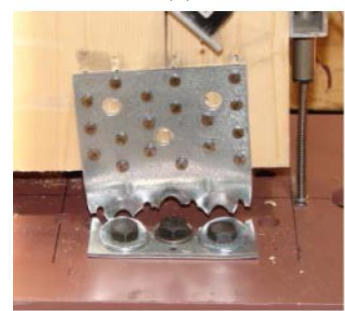

(c)

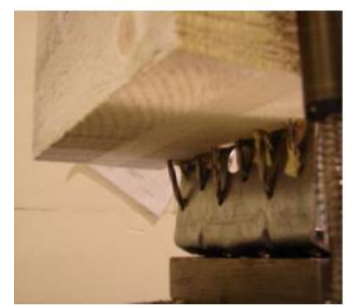

(e)

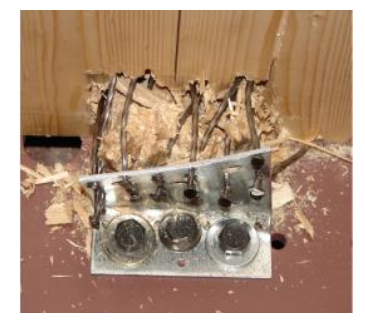

(b)

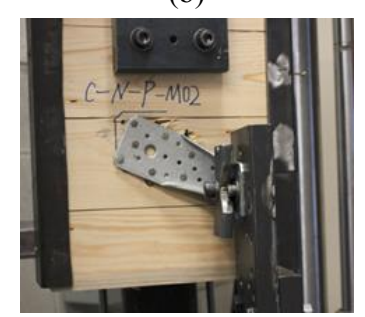

(d)

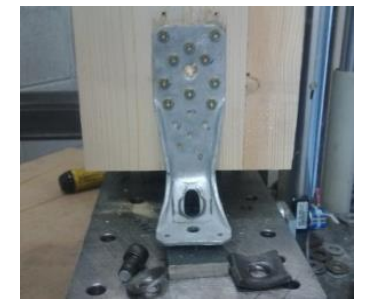

(f)
Fig.6. Failure modes of bracket anchor connections:(1) fasteners pulling out; (2) block failure; (3)tension fracture of the angle bracket; (4) Severe deformation of the bracket; (5) CLT edge failure; (6)bolt abruption.

Failure mode (b): the pull-out resistance from bond strength and friction between fasteners and CLT block is higher than the tensile strength of the CLT 
block but lower than the strength of the bracket, block crush failure will occur suddenly. It is a nonpredictable brittle failure mode. In addition, the failure mode depends also on defects in wood fibers. However, this failure mode should be avoided because block brittle failure destroys the CLT wall.

Failure mode (c) and Failure mode (d): These failure modes are concentrated on bracket itself. The pull-out resistance from bond strength and friction between fasteners and CLT block is high enough and the wood remains intact, the right angle part of the connections are weakest and most dangerous section. Tension resistance around the corner of Bracket A is not enough to lead to failure mode(c). And shear stiffness around the angle part of Bracket $\mathrm{C}$ is not enough to coordinate fasteners embedded movement in failure mode (d), which results in severe deformation, bending and torsion of the angle part of the bracket. Then, warping stress produced from angle part of the bracket is very harmful to the threads of anchor bolts and it can destroy the anchor system. However, both failure modes are ductile failure, but do not make full use of the most resistance to dissipate energy.

Failure mode (e): due to low anchorage position, the edge of CLT block was pulled through. Hence, improper construction leads to edge failure of CLT block and reasonable edge distance of anchorage should be considered.

Failure mode (f): the anchoring bolt was tension abruption due to improper anchor system design. The slip load is concentrated on one bolt near the angle of bracket to resistance, while another bolt far from the angle of bracket didn't play the anchoring role sufficiently. However, this failure mode should be avoided because bolt tension abruption failure is brittle and it is hard to replace it.

\subsection{Mechanical properties analysis}

Fasteners pull-out failure is recommended as an ideal failure mode to find reasonable combinations of brackets and fasteners. Afterwards, there are seven kinds of bracket anchor connections to be found as follows:

Table 1. The types of combination of brackets and fasteners

\begin{tabular}{|c|c|c|}
\hline $\begin{array}{c}\text { Types of } \\
\text { connection }\end{array}$ & $\begin{array}{c}\text { Types of } \\
\text { angle bracket }\end{array}$ & Types of fasteners \\
\hline Connection 1 & Bracket A & 18 nails $16 \mathrm{~d} \times 3 \frac{1}{2}$ " $^{\prime}$ \\
\hline Connection 2 & Bracket A & 9 screws $5 \times 90 \mathrm{~mm}$ \\
\hline Connection 3 & Bracket A & 18 nails $4 \times 70 \mathrm{~mm}$ \\
\hline Connection 4 & Bracket B & 10 screws $16 \mathrm{~d} \times 31 / 2 "$ \\
\hline Connection 5 & Bracket C & 7 nails $16 \mathrm{~d} \times 31 / 2 "$ \\
\hline Connection 6 & Bracket C & 7 screws $5 \times 90 \mathrm{~mm}$ \\
\hline Connection 7 & Bracket C & 9 screws $4 \times 70 \mathrm{~mm}$ \\
\hline
\end{tabular}

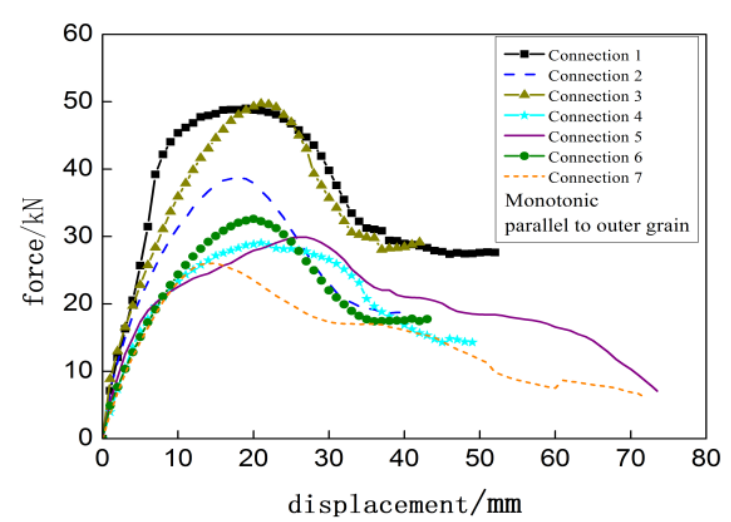

Fig.7. Monotonic backbone curves of different connections in parallel to outer grain direction

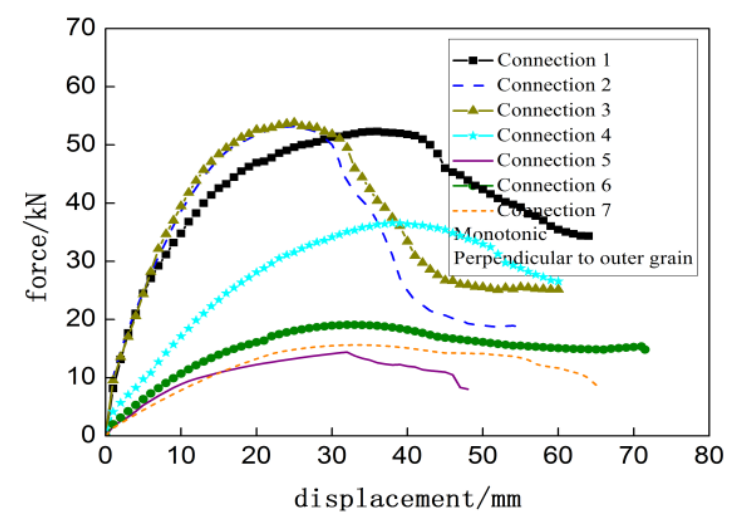

Fig.8. Monotonic backbone curves of different connections in perpendicular to outer grain direction

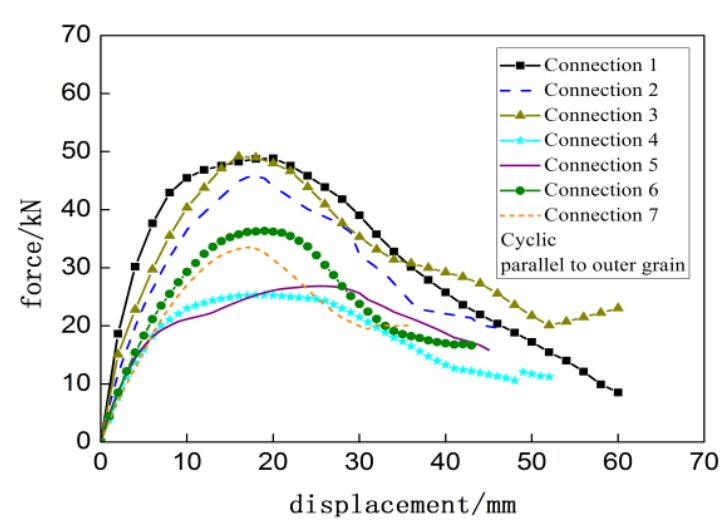

Fig.9. Cyclic backbone curves of different connections in parallel to outer grain direction

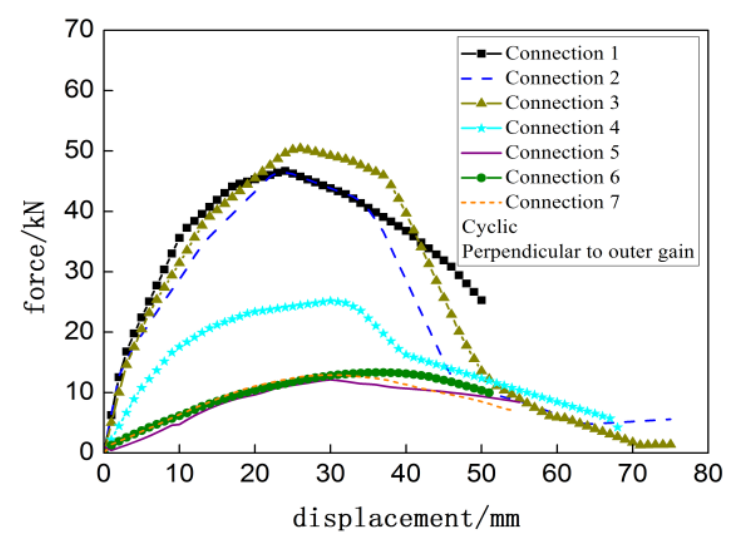


Fig.10. Cyclic backbone curves of different connections in perpendicular to outer grain direction

Three monotonic loading tests and three cyclic loading tests are performed in each direction of one kind of anchor connection, and mean monotonic and hysteretic backbone curves are obtained as follows(Fig.7-10).

It is showed that there is no obvious yield point with highly non-linear about each backbone curves of connections. Monotonic and hysteretic envelope curves (both for parallel and perpendicular to outer grain direction test) computed based on equivalent energy elastic-plastic (EEEP) are summarized in Table2 and 3. The parameters used to define the envelopes are:

- $\mathrm{K}_{\mathrm{e}}$ is equivalent elastic stiffness.
- $\quad \mathrm{P}_{\text {peak }}$ is the peak load of the envelope curve.

- $\Delta_{\text {peak }}$ is the corresponding displacement at the peak load.

- $\quad \mathrm{P}_{\mathrm{u}}$ is defined to the ultimate load corresponding to failure limit state which is at $80 \%$ of the peak load.

- $\Delta_{\mathrm{u}}$ is the corresponding displacement at the failure load $\left(\mathrm{P}_{\mathrm{u}}\right)$.

- $\quad \mathrm{P}_{\mathrm{y}}$ is equivalent yield load but actually there is no significant yield point on the hysteretic envelope curve.

- $\Delta_{\mathrm{y}}$ is the displacement at the yield load.

- $\mathrm{D}$ is ductility factor defined as the ratio of the ultimate displacement $\left(\Delta_{\mathrm{u}}\right)$ and the yield displacement $\left(\Delta_{\mathrm{y}}\right)$.

Table 2 Mechanical parameters estimation of bracket connections in parallel to outer grain direction

\begin{tabular}{|c|c|c|c|c|c|c|c|c|c|}
\hline $\begin{array}{c}\text { Connection } \\
\text { type }\end{array}$ & $\begin{array}{c}\text { Loading } \\
\text { type }\end{array}$ & $\mathrm{K}_{\mathrm{e}}(\mathrm{kN} / \mathrm{mm})$ & $\mathrm{P}_{\mathrm{y}}(\mathrm{kN})$ & $\mathrm{P}_{\text {peak }}(\mathrm{kN})$ & $\mathrm{P}_{\mathrm{u}}(\mathrm{kN})$ & $\Delta_{\mathrm{y}}(\mathrm{mm})$ & $\Delta_{\text {peak }}(\mathrm{mm})$ & $\Delta_{\mathrm{u}}(\mathrm{mm})$ & $\mathrm{D}$ \\
\hline \multirow{2}{*}{1} & monotonic & 5.19 & 46.7 & 49.01 & 39.21 & 9.06 & 19 & 30.5 & 3.37 \\
\cline { 2 - 12 } & cyclic & 9.08 & 44.6 & 48.9 & 39.1 & 5.4 & 20 & 30 & 5.56 \\
\hline \multirow{2}{*}{2} & monotonic & 4.9 & 34.1 & 38.7 & 31.0 & 6.9 & 18 & 24 & 3.48 \\
\cline { 2 - 12 } & cyclic & 5.1 & 40.2 & 45.8 & 36.6 & 7.9 & 18 & 29 & 3.67 \\
\hline \multirow{2}{*}{3} & monotonic & 4.9 & 44 & 49.7 & 39.8 & 9.0 & 21.0 & 27.9 & 3.1 \\
\cline { 2 - 12 } & cyclic & 6.2 & 43.6 & 49.2 & 39.3 & 7.0 & 16 & 27 & 3.86 \\
\hline \multirow{2}{*}{4} & monotonic & 3.5 & 26.2 & 29.1 & 23.2 & 7.5 & 21 & 33.9 & 4.52 \\
\cline { 2 - 11 } & cyclic & 3.7 & 23.4 & 25.3 & 20.3 & 6.3 & 19 & 31.4 & 4.98 \\
\hline \multirow{2}{*}{5} & monotonic & 4.26 & 25.94 & 29.93 & 23.95 & 6.09 & 26.5 & 34 & 5.58 \\
\cline { 2 - 11 } & cyclic & 3.9 & 23.88 & 26.85 & 21.48 & 6.12 & 25.5 & 35.55 & 5.81 \\
\hline \multirow{2}{*}{6} & monotonic & 3.26 & 28.48 & 32.6 & 26.09 & 8.74 & 20 & 27.2 & 3.11 \\
\cline { 2 - 11 } & cyclic & 3.91 & 33.38 & 36.36 & 29.09 & 8.54 & 19 & 26.75 & 3.13 \\
\hline \multirow{2}{*}{7} & monotonic & 3.16 & 23.48 & 26.03 & 20.83 & 7.43 & 14.5 & 23.5 & 3.16 \\
\cline { 2 - 10 }$y$ & cyclic & 3.16 & 30.57 & 33.63 & 26.90 & 9.67 & 17.5 & 23.5 & 2.4 \\
\hline
\end{tabular}

Table 3 Mechanical parameters estimation of bracket connections in perpendicular to outer grain direction

\begin{tabular}{|c|c|c|c|c|c|c|c|c|c|}
\hline $\begin{array}{c}\text { Connection } \\
\text { type }\end{array}$ & $\begin{array}{c}\text { Loading } \\
\text { type }\end{array}$ & $\mathrm{K}_{\mathrm{e}}(\mathrm{kN} / \mathrm{mm})$ & $\mathrm{P}_{\mathrm{y}}(\mathrm{kN})$ & $\mathrm{P}_{\text {peak }}(\mathrm{kN})$ & $\mathrm{P}_{\mathrm{u}}(\mathrm{kN})$ & $\Delta_{\mathrm{y}}(\mathrm{mm})$ & $\Delta_{\text {peak }}(\mathrm{mm})$ & $\Delta_{\mathrm{u}}(\mathrm{mm})$ & $\mathrm{D}$ \\
\hline \multirow{2}{*}{1} & monotonic & 5.28 & 47 & 52.37 & 41.9 & 8.9 & 36 & 50.5 & 5.67 \\
\cline { 2 - 11 } & cyclic & 5.1 & 41.5 & 46.7 & 37.4 & 8.1 & 24 & 39.2 & 4.84 \\
\hline \multirow{2}{*}{2} & monotonic & 4.5 & 41 & 45.6 & 36.5 & 9.1 & 23 & 32 & 3.51 \\
\cline { 2 - 11 } & cyclic & 4.2 & 40.2 & 46.4 & 37.1 & 9.6 & 24 & 36.7 & 3.82 \\
\hline \multirow{2}{*}{3} & monotonic & 4.3 & 42.2 & 46.1 & 36.9 & 9.7 & 25 & 34.7 & 3.58 \\
\cline { 2 - 10 } & cyclic & 4.1 & 44.5 & 50.4 & 40.3 & 10.8 & 40.3 & 39.7 & 3.68 \\
\hline \multirow{2}{*}{4} & monotonic & 1.5 & 28.3 & 31.3 & 25.1 & 18.6 & 38 & 54.1 & 2.91 \\
\cline { 2 - 10 } & cyclic & 2.2 & 22.5 & 25.2 & 20.1 & 10.3 & 30 & 36.7 & 3.56 \\
\hline \multirow{2}{*}{5} & monotonic & 0.87 & 11.03 & 12.35 & 9.88 & 12.68 & 32 & 50.25 & 3.96 \\
\cline { 2 - 10 } & cyclic & 0.48 & 11.21 & 12.12 & 9.70 & 23.35 & 30 & 48 & 2.06 \\
\hline \multirow{2}{*}{6} & monotonic & 1.02 & 14.51 & 16.35 & 13.08 & 14.23 & 33 & 57.5 & 4.04 \\
\cline { 2 - 10 } & cyclic & 0.64 & 12.17 & 13.3 & 10.64 & 19 & 36.5 & 49.25 & 2.60 \\
\hline \multirow{2}{*}{7} & monotonic & 0.70 & 13.15 & 13.4 & 10.72 & 18.8 & 33.5 & 54.5 & 2.90 \\
\cline { 2 - 9 } & cyclic & 0.67 & 11.8 & 12.9 & 10.32 & 17.6 & 30 & 43.4 & 2.46 \\
\hline
\end{tabular}

Overall, according to Table 2-3, most ductility factors of bracket connections for monotonic and cyclic or for parallel or perpendicular to outer gain direction are above 3.0. Furthermore, most ductility factors of backbone curves from cyclic tests are higher than monotonic tests, that's because ductility 
mechanism give full play and the deformation capacity of bracket connections strengthen in cyclic tests.

In terms of parallel to outer grain direction test including monotonic and cyclic loading, seen from Table2, Connection 1, 2 and 3 based on Bracket $\mathrm{A}$ has the greatest $\mathrm{P}_{\text {peak }}$ values, between $38 \sim 49.7 \mathrm{kN}$; next is Connection 5,6 and 7 based on Bracket C, between $26.85 \sim 36.36 \mathrm{kN}$; the last is Connection 4 based on Bracket B, between $25.3 \sim 29.1 \mathrm{kN}$. For cyclic loading among three kinds of connections based on Bracket A, Connection 1 has the greatest $\mathrm{K}_{\mathrm{e}}$ value $(9.08 \mathrm{kN} / \mathrm{mm})$, next is Connection $3(6.2 \mathrm{kN} / \mathrm{m})$, and the last is Connection $2(5.1 \mathrm{kN} / \mathrm{m})$; Similar trend are observed for $\mathrm{P}_{\mathrm{y}}$ values; in addition, Connection 1 and 3 have higher $\mathrm{P}_{\text {peak }}$ values $(48.9 \mathrm{kN}, 49.2 \mathrm{kN})$ than Connection $3(45.8 \mathrm{kN})$. It is noted that Connection 1, 2 and 3 based on Bracket A have relatively high resistance for loadslip and Connection 1 shows higher ductility ratio( $(D=5.56)$ than Connection $2 \quad(D=3.86)$ and Connection 3( $\mathrm{D}=3.67)$.

For perpendicular to outer grain direction tests including monotonic and cyclic loading, seen from Table3, Connection 1, 2 and 3 based on Bracket A achieve the highest $\mathrm{P}_{\text {peak }}$ values, between 46.1 52.37 $\mathrm{kN}$; next is Connection 4 base on Bracket $\mathrm{B}$, between 25.2 31.3kN; the rest including Connection 5, 6, 7, 8 based on Bracket $\mathrm{C}$, have the smallest values between $12.12 \sim 16.35 \mathrm{kN}$. Comparing to parallel and perpendicular to outer grain direction tests, although tension resistance of Bracket $\mathrm{C}$ in parallel to outer grain direction test is good, weak shear stiffness $\left(\mathrm{K}_{\mathrm{e}}=0 \sim 1.02 \mathrm{kN} / \mathrm{mm}\right)$ and narrow angle part of Bracket $\mathrm{C}$ cause severe deformation and warping stress from bending and torsion of the angle part of the bracket, particularly subjected to cyclic loading. Under the situation, it is shown that there is no obvious decent stage after reaching peak load on cyclic backbone curves. Based on above calculation, Connection 1 still has the highest $\mathrm{K}_{\mathrm{e}}$ value of $5.1 \mathrm{kN} / \mathrm{mm}$, followed by Connection $2(4.2 \mathrm{kN} / \mathrm{mm})$ and Connection 3 (4.1 $\mathrm{kN} / \mathrm{mm}$ ) and the rest with the smaller values under cycling loading situation. Meanwhile, Connection 3 achieves the greatest $P_{\text {peak }}$ value $(50.3 \mathrm{kN})$, next is Conncetion $1(46.7 \mathrm{kN})$ and the third is Connection 2 (46.4 kN) subject to cyclic loading. However, in terms of ductility ratio, Connection 1 shows highest ductility ratio $(D=4.84)$, the second is Connection $2(D=3.82)$, the third is Connection $3(\mathrm{D}=3.68)$ and the rest with smaller value. Above all, the distribution of tension and shear stiffness of Connection 1, 2, 3 based Bracket A is proper, the connections based Bracket A can provide high tension and shear resistance. In addition, Connection 1 has an excellent ductility and load capacity among seven kinds of bracket anchor connections.

\section{Conclusions}

(1) Various fasteners and bracket types have large effect on failure modes and mechanical behavior of bracket anchor connections. The initial tests are necessary which helped to define the number of fasteners to each type of bracket and ideal failure modes.

(2) Fasteners pull-out failure for bracket anchor connections is an ideal ductile failure mode as the most energy can be dissipated by forming plastic hinges of fasteners while not destroying bracket and the CLT block. According to monotonic and cyclic backbone curves, it firstly experienced elastic-plastic stage but there is no obvious yield point; then has certain deformation to reach the peak load; afterwards, from peak load to fails there is an obvious but ductile decent stage.

(3) The distribution of tension and shear stiffness of Bracket A and B in both directions are suitable, which can coordinate fasteners movement embedded in CLT. Proper combination of fasteners and Bracket A can provide an ideal failure mode, high tension and shear capacity $(38 \sim 52.37 \mathrm{kN})$ and ductility; next is Bracket B, which can offer relative high tension and shear force $(25.3 \sim 31.3 \mathrm{kN})$ in both direction; although relative high tension resistance capacity of Bracket $\mathrm{C}$ $(26.85 \sim 36.36 \mathrm{kN})$, weak shear stiffness $\left(\mathrm{K}_{\mathrm{e}}=0 \sim 1.02\right.$ $\mathrm{kN} / \mathrm{mm})$ and shear resistance capacity $(12.12 \sim 16.35 \mathrm{kN})$ around the angle of Bracket $\mathrm{C}$ can result in severe deformation, bending and torsion of the bracket itself. In addition, Connection 1 based on Bracket $\mathrm{A}$ has an excellent ductility and load capacity among seven kinds of bracket anchor connections.

\section{References}

1. A. Ceccotti, M. Follesa, M. P. Lauriola, et al. Sofie Project-Test Results on the Lateral Resistance of Cross-Laminated Wooden Panels. Proceedings of First European Conference on Earthquake Engineering and Seismology(2006)

2. B. Dujic, J. Pucelj, R. Zarnic. Testing of Racking Behavior of Massive Wooden Wall Panels. Proceedings of the 37 th CIB-W18 Meeting, 37-15-2:1-10(2004)

3. M. Popovski, J. Schneider, M. Schweinsteiger. Lateral load resistance of cross-laminated wood panels. Proceedings of 11th WCTE(2010)

4. M. Fragiacomo, B. Dujic, I. Sustersic. Elastic and ductile design of multi-storey crosslam massive wooden buildings under seismic actions, Eng. Struct. 33(11): 3043-3053(2011)

5. Y. L. Shen, J. Schneider, S. Tesfamariam, et al. Hysteresis behavior of bracket connection in crosslaminated-timber shear walls, Constr. Build. Mater., 48: 980-991(2013)

6. J. Schneider. Connections in Cross-LaminatedTimber Shear Walls considering the Behaviour under monotonic and cyclic lateral Loading [D]. Stuttgart; University of Stuttgart(2009)

7. I. Gavric, M. Fragiacomo, A. Ceccotti. Cyclic behaviour of typical metal connectors for cross- 
laminated (CLT) structures. Eur. J. Wood Prod.,73(2): 179-191(2015)

8. A. Polastri, I. Giongo, A. Angeli, et al. Mechanical characterization of a pre-fabricated connection system for cross laminated timber structures in seismic regions . Eng. Struct.,167:705-715(2017)

9. A. Polastri, A. Angeli. An innovative connection system for CLT structures: Experimentalnumerical analysis. Proceedings of the 13st WCTE (2014)

10. A. Kraler, J. Kögl, R. Maderebner, et al.SherpaCLT-Connector for Cross Lamniated Timber(CLT)
Elements. Proceedings of the 13st WCTE(2014)

11. American National Standards Institute. ANSI/APA Standard for Performance-Rated Cross-Laminated Timber.Washington: APA-The Engineered Wood Association(2012)

12. ASTM E2126-11. Standard Test Methods for cyclic(Reversed) Load Test for Shear Resistance of Walls for Buildings(2011)

13. H. Krawinkler, F. Parisi, L. Ibarra, et al. Development of a testing protocol for woodframe structures. CUREE Richmond, CA.(2001) 